\title{
Age and tissue specific differences in the development of acute insulin resistance following injury
}

\author{
Lidong Zhai ${ }^{1}$ and Joseph L Messina ${ }^{1,2}$ \\ ${ }^{1}$ Division of Molecular and Cellular Pathology, Department of Pathology, The University of Alabama at Birmingham, 1670 University Boulevard, Birmingham, \\ Alabama 35294-0019, USA \\ ${ }^{2}$ Veterans Affairs Medical Center, Birmingham, Alabama 35233, USA \\ (Correspondence should be addressed to J L Messina at Division of Molecular and Cellular Pathology, Department of Pathology, The University of Alabama \\ at Birmingham; Email: messinaj@uab.edu)
}

\begin{abstract}
Injuries, hemorrhage, sepsis, burn, and critical illnesses all induce insulin resistance, and insulin resistance is strongly associated with advancing age. However, the effect of age on injury induced insulin resistance is not well studied. We performed surgical trauma in male rats of three different ages (3-, 6-, and 10-weeks old). Rats were either hemorrhaged to a mean arterial pressure of $35-40 \mathrm{mmHg}$ and subsequently maintained at that pressure for up to $90 \mathrm{~min}$, or maintained without hemorrhage as controls. Results indicate that insulininduced intracellular signaling was diminished in liver and skeletal muscle of 6- and 10-week old rats following trauma and hemorrhage. In even younger rats, immediately postweaning ( $\sim 3$ weeks of age), insulin signaling was lost in liver, but not in skeletal muscle. Glucocorticoids can play a role in the chronic development of insulin resistance. Our results
\end{abstract}

demonstrate that corticosterone levels were increased in 6- and 10-week old animals following hemorrhage, but little change was measured in 3-week old animals. Blockade of glucocorticoid synthesis prevented the development of insulin resistance in skeletal muscle, but not in liver of 6- and 10-week old rats. Moreover, skeletal muscle glucocorticoid receptor levels increased dramatically between 3 and 6 weeks of age. These results indicate that trauma and hemorrhage-induced hepatic insulin resistance occurs at all ages tested. However, there is no development of insulin resistance following trauma and hemorrhage in skeletal muscle of post-weaning rats. In skeletal muscle of 6- and 10 -week old rats, inhibition of glucocorticoid levels prevents the development of insulin resistance.

Journal of Endocrinology (2009) 203, 365-374

\section{Introduction}

Acute insulin resistance is common following trauma, hemorrhage, sepsis, burn, and critical illness (Ikezu et al. 1997, Carter 1998, Van den Berghe et al. 2001, Cree \& Wolfe 2008). Chronic insulin resistant states, such as Type 2 diabetes, hypertension, cardiovascular disease, and the metabolic syndrome are all associated with advancing age (Meneilly \& Tessier 1995, Alexander 2003, McEniery et al. 2007). However, little is known about the effects of age on the development of insulin resistance following injuries or critical illness, and whether the degree of insulin resistance varies with age. Injury and critical illness-induced hyperglycemia, sometimes referred to as 'critical illness diabetes', may increase mortality and morbidity (Van den Berghe et al. 2001, Bochicchio et al. 2005, Langouche et al. 2005). Hyperglycemia occurs frequently in critically ill children and adults (Garcia et al. 2007, Klein et al. 2007), and can be readily explained by a decrease of peripheral glucose disposal in muscle, or increased hepatic glucose production, often coincident with decreased responses to insulin (insulin resistance). Intensive insulin therapy of critically ill adult patients in the surgical intensive care unit may reduce morbidity and mortality (Van den Berghe et al. 2001, Hansen et al. 2003). However, there is some controversy since recent studies suggest that intensive insulin therapy may not be as beneficial (Arabi et al. 2008, Braithwaite 2009). In addition, intensive insulin therapy increases the incidence of hypoglycemic episodes with the potential for negative outcomes (Finfer et al. 2009). Thus, an understanding of the molecular mechanisms of tissue-specific acute insulin resistance may provide new therapeutic approaches in treating the critically ill following injury.

Insulin regulates glucose metabolism by binding to the insulin receptor (IR) on the surface of target cells. Binding of insulin to the IR leads to activation of its tyrosine kinase activity which promotes association and phosphorylation of intracellular IR substrate proteins (IRSs), which act as docking proteins in IR/phosphatidylinositol 3 kinase (PI3K)/AKT pathway (White 1997, Nakae \& Accili 1999). Reduced signaling through this pathway can cause insulin resistance. Previous data from our laboratory indicate that trauma and hemorrhage induces the rapid development of insulin resistance in the liver and skeletal muscle of adult rats 
(Ma et al. 2003, 2004, Thompson et al. 2008, Xu et al. 2008, Li et al. 2009), but it is not known whether there is a difference in the development of acute insulin resistance at different ages following injury.

Glucocorticoids are a potential causative factor in chronic and acute insulin resistance (Witchel \& DeFranco 2006, Qi \& Rodrigues 2007, Vegiopoulos \& Herzig 2007, Li et al. 2009) by interfering with components of the insulin signaling cascade (Giorgino et al. 1993, Saad et al. 1993). For the present work, we hypothesized that glucocorticoids play a role in the development of acute insulin resistance in rats following trauma and hemorrhage, and that this role may differ at different ages since the hypothalamic/pituitary/ adrenal axis changes with age. We found that trauma and hemorrhage induced insulin resistance in the liver and skeletal muscle of both 6- and 10-week old rats. However, in 3-week old rats, trauma and hemorrhage induced insulin resistance in the liver, but not in skeletal muscle. Glucocorticoids may play an important role in the hemorrhage-induced insulin resistance in skeletal muscle of 6- and 10-week old animals.

\section{Materials and Methods}

\section{Animal model of trauma and hemorrhage}

All procedures were performed in accordance with the guidelines set forth in the Animal Welfare Act and the Guild for the Care and Use of Laboratory Animal by the National Institutes of Health. The experimental protocol was approved by the Institutional Animal Care and Use Committee of the University of Alabama at Birmingham. Three different ages of male Sprague-Dawley rats ( 3-, 6-, and 10-weeks old; 3-5 animals/group) were fasted $18-20 \mathrm{~h}$ prior to surgery, but were allowed access to water ad libitum. Rats of $\sim 10-12$ weeks old are most common in studies of the rat trauma and hemorrhage model (Ma et al. 2003, 2004, Thompson et al. 2008, Xu et al. 2008). Rats of 6-8 weeks old are most common in the study of insulin sensitivity, since as rats age past this point, they become less responsive to insulin. Our previous work (Li et al. 2009) indicates that glucocorticoids play a key role in skeletal muscle insulin resistance around 10-11 week old rats following trauma and hemorrhage. Numerous studies indicate changes in the levels of glucocorticoids in response to stress, but this may not be true in very young rats, prior to weaning. Thus, we chose the three age groups to study the development of insulin resistance following injury. A rat model of injury, labeled trauma and hemorrhage, as previously described (Ma et al. 2003, 2004, Thompson et al. 2008, Xu et al. 2008, Li et al. 2009), was used in this study. Briefly, at room temperature, anesthesia was by isoflurane inhalation, and a ventral midline laparotomy $(3,4$, or $5 \mathrm{~cm}$, based on the age of the rat) was performed representing soft tissue trauma, and then the abdomen was closed. The polyethylene-50 catheters (Becton Dickinson Intramedic, Sparks, MD, USA) that were placed in the right and left femoral arteries and the right femoral vein, for bleeding and monitoring of mean arterial pressure. Rats were bled rapidly to a mean arterial pressure of $35-40 \mathrm{mmHg}$ within $10 \mathrm{~min}$. Once mean arterial pressure reached $40 \mathrm{mmHg}$, the timing of the hemorrhage period began. Small amounts of additional blood was withdrawn or saline was supplied to each animal to maintain a mean arterial pressure of $35-40 \mathrm{mmHg}$ for the $90 \mathrm{~min}$ (TH90') hemorrhage period.

\section{Study design}

Due to the considerable trauma incurred during anesthesia, catheterization and opening of the abdominal cavity to perform the insulin injections (see below), it was impossible to have a completely trauma-free control group. Thus, the baseline animals chosen in these experiments were the trauma alone rats $\left(\mathrm{T}^{\prime}\right)$ that were subjected to anesthesia, laparotomy, catheterization, and then killed immediately following saline or insulin injection. Other trauma alone groups were subjected to the same procedures but killed at $90 \mathrm{~min}$ $\left(\mathrm{T}^{\prime} 0^{\prime}\right)$ after catheterization. The trauma and hemorrhage groups were subjected to the same procedures as the trauma groups, but followed by hemorrhage and subsequently killed at $90 \mathrm{~min}$, the end of the hemorrhage period $\left(\mathrm{TH} 90^{\prime}\right)$.

\section{Drug administration}

Metyrapone was purchased from Sigma and was dissolved in propylene glycol. For metyrapone pretreatment, rats received one injection of metyrapone $(150 \mathrm{mg} / \mathrm{kg}$, i.p.) or vehicle $3 \mathrm{~h}$ prior to the surgery, a time and dose known to inhibit the stress-induced increase in plasma corticosterone levels (Li et al. 2009).

\section{Tissue and blood harvesting procedures}

At the $0^{\prime}$ or $90^{\prime}$ time points, the abdominal cavity of the animal was opened, the portal vein was exposed, and insulin $(5 \mathrm{U})$ or saline was injected into the portal vein. One minute after the injection, liver, triceps, and extensor digitorum longus (EDL) were removed and snap frozen in liquid nitrogen for future analysis. Immediately before insulin or saline injection, blood was drawn from the inferior vena cava and centrifuged at $5000 \boldsymbol{g}$ for $10 \mathrm{~min}$. The plasma was stored at $-80{ }^{\circ} \mathrm{C}$ for future use. Corticosterone levels were determined using a RIA kit (MP Biomedicals, Orangeburg, NY, USA) according to the manufacturer's instructions by a core facility of the UAB Clinical Nutrition Research Center.

\section{Western immunoblot analysis}

Liver and muscle tissues from each animal $(0 \cdot 2 \mathrm{~g})$ were homogenized in extraction buffer, as described previously, and stored at $-80^{\circ} \mathrm{C}$ until use (Ma et al. 2003, 2004, Xu et al. 2008). The protein concentration of tissue lysates were determined by the BCA method (Pierce, Rockford, IL, USA). 
For immunoblotting, $30 \mu \mathrm{g}$ protein per lane was resolved by $10 \%$ SDS-PAGE and transferred to nitrocellulose paper. The western transfers were immunoblotted with the following primary antibodies: anti-phospho-Y972-IR, antiphospho-Y612-IRS-1 (Invitrogen Biosource International), anti-total IR $\beta$ (Santa Cruz Biotechnology, Santa Cruz, CA, USA), anti-total glucocorticoid receptor (GR), anti-phosphoS473-AKT, anti-total AKT, and anti-total ERK (Cell Signaling Technology, Danvers, MA, USA). HRP-conjugated secondary antibody (Cell Signaling Technology) was then added for detection of bound antibody by enhanced chemiluminescence (Amersham Pharmacia Biotech) or Supersignal Femto Maximum Sensitivity Substrate reagent (Pierce Biotechnology). The blots were stripped using Reblot reagent (CHEMICON International,Inc., Temecula, CA, USA) for $15 \mathrm{~min}$, and then re-probed with a different antibody.

\section{Densitometric and statistical analysis}

Enhanced chemiluminescent images of immunoblots were scanned and quantified using Zero D-Scan (Scanalytics Corp., Fairfax, VA, USA). All data were analyzed by
ANOVA by Tukey-Kramer Multiple Comparisons Test or Student's $t$-test using the InStat statistical program by GraphPad Software, Inc (San Diego, CA, USA).

\section{Results}

Trauma and hemorrhage-induced decreases in insulin signaling $(P-A K T)$ : differences evident in triceps of 3-week old rats

We previously found that insulin signaling in liver and skeletal muscle was only minimally affected by trauma alone, but the combination of trauma and hemorrhage abolished insulin signaling in both liver and skeletal muscle in adult rats (Ma et al. 2003, 2004, Thompson et al. 2008, $\mathrm{Xu}$ et al. 2008, Li et al. 2009). In the present study, we first examined insulin signaling following trauma and hemorrhage in animals at different ages (3-, 6-, and 10-weeks old) by determining insulin-induced serine phosphorylation of $\mathrm{AKT}$ (P-AKT (S473)), a process that is required for AKT activation (Hill et al. 2002). We found that insulin-induced hepatic P-AKT was completely lost in animals at all three
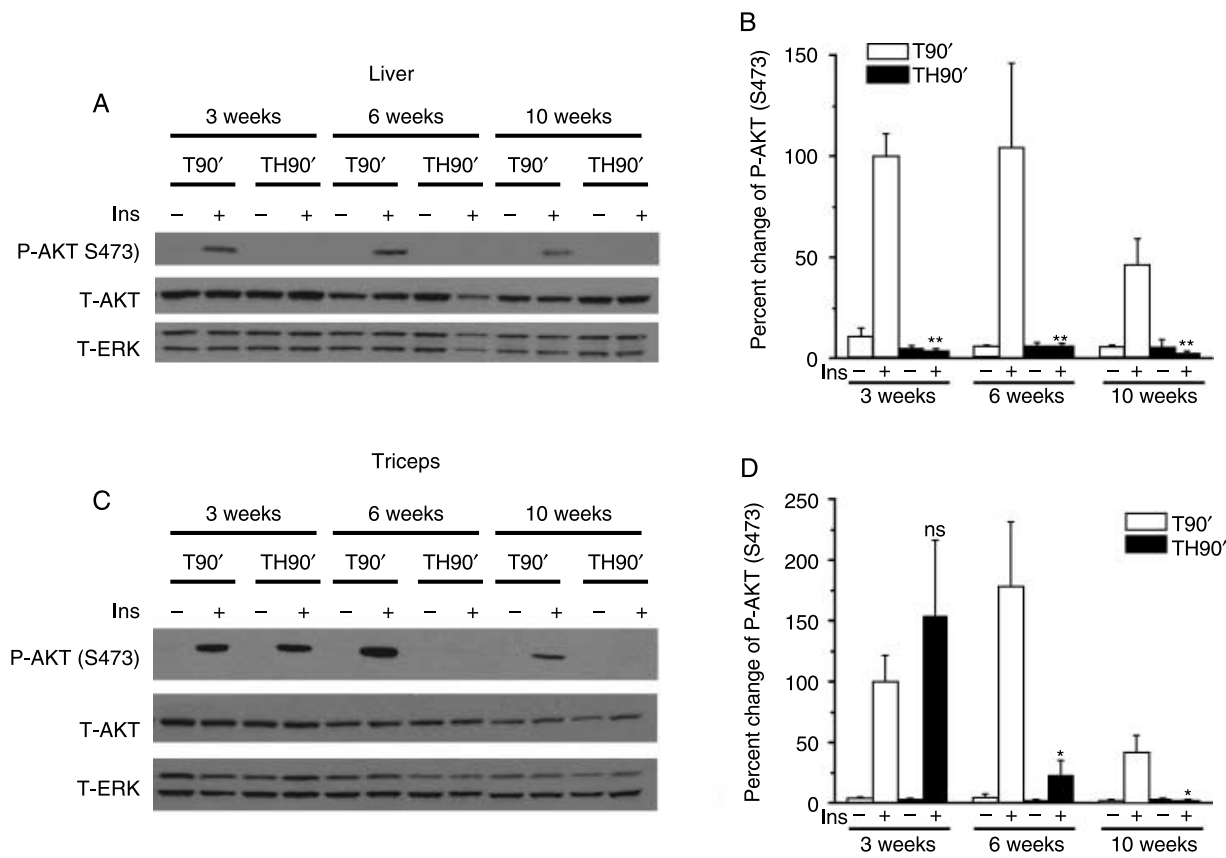

Figure 1 Hepatic and skeletal muscle insulin signaling via serine phosphorylation of AKT at different ages following trauma and hemorrhage. Rats were subjected to trauma alone $\left(\mathrm{T}^{\prime} 0^{\prime}\right)$ or trauma and hemorrhage $\left(\mathrm{TH}^{\prime}\right)$. At $90^{\prime}$ (with or without hemorrhage) either saline (-) or $5 \mathrm{U}$ insulin (Ins; +) was injected via the portal vein. The liver and triceps were removed after $1 \mathrm{~min}$ and frozen in liquid nitrogen. (A and C) Tissue lysates from liver and triceps were subjected to western blotting with antibodies specific for phospho-AKT (P-AKT (S473)), total AKT (T-AKT), and total ERK (T-ERK). (B and D) Autoradiographs were quantified by scanning densitometry. Data are presented as the mean \pm S.E.M. of the percentage change of P-AKT (S473) by insulin of three rats in each group. The phosphorylated protein levels in $\mathrm{T}^{\prime} 0^{\prime}$ ( 3 weeks) with insulin treatment were arbitrarily set to $100 \%$. In this and all the following figures: ${ }^{*} P<0 \cdot 05$; ${ }^{*} P<0 \cdot 01$; ${ }^{* *} P<0 \cdot 001$; ns, not significant, compared with $\mathrm{T} 90^{\prime}$ with insulin injection of the same age group. 
ages studied (3-, 6-, and 10-weeks old) $90 \mathrm{~min}$ following trauma and hemorrhage (TH90'; Fig. $1 \mathrm{~A}$ and $\mathrm{B})$. This indicates that trauma and hemorrhage abolished hepatic insulin signaling independent of age.

We next examined insulin-induced P-AKT in skeletal muscle following trauma and hemorrhage at different ages and consistent with our previous findings in adult animals, in 10-week old rats (Thompson et al. 2008), insulininduced P-AKT (S473) was severely diminished in triceps following trauma and hemorrhage $\left(\mathrm{TH}^{\prime} \mathrm{O}^{\prime}\right)$ compared with trauma alone (T90'; Fig. $1 \mathrm{C}$ and D). The large decrease of insulin-induced P-AKT (S473) was also observed at
6 weeks of age. However, there was little change in insulin-induced P-AKT (S473) in triceps following trauma and hemorrhage from 3 -week old rats (Fig. 1C and D). This suggests something different about the response of skeletal muscle in the youngest rats studied. Although there were observed decreases in the levels of P-AKT (S473) induced by insulin in trauma only animals $\left(\mathrm{T}^{\prime} 0^{\prime}\right)$ in the liver and triceps from the oldest animals (Fig. 1B and D), the fold-induction by insulin did not reach statistical significance by ANOVA. Moreover, there was no significant difference in the total amounts of the AKT or ERK1/2 proteins in the three age groups (Fig. 1A and C).
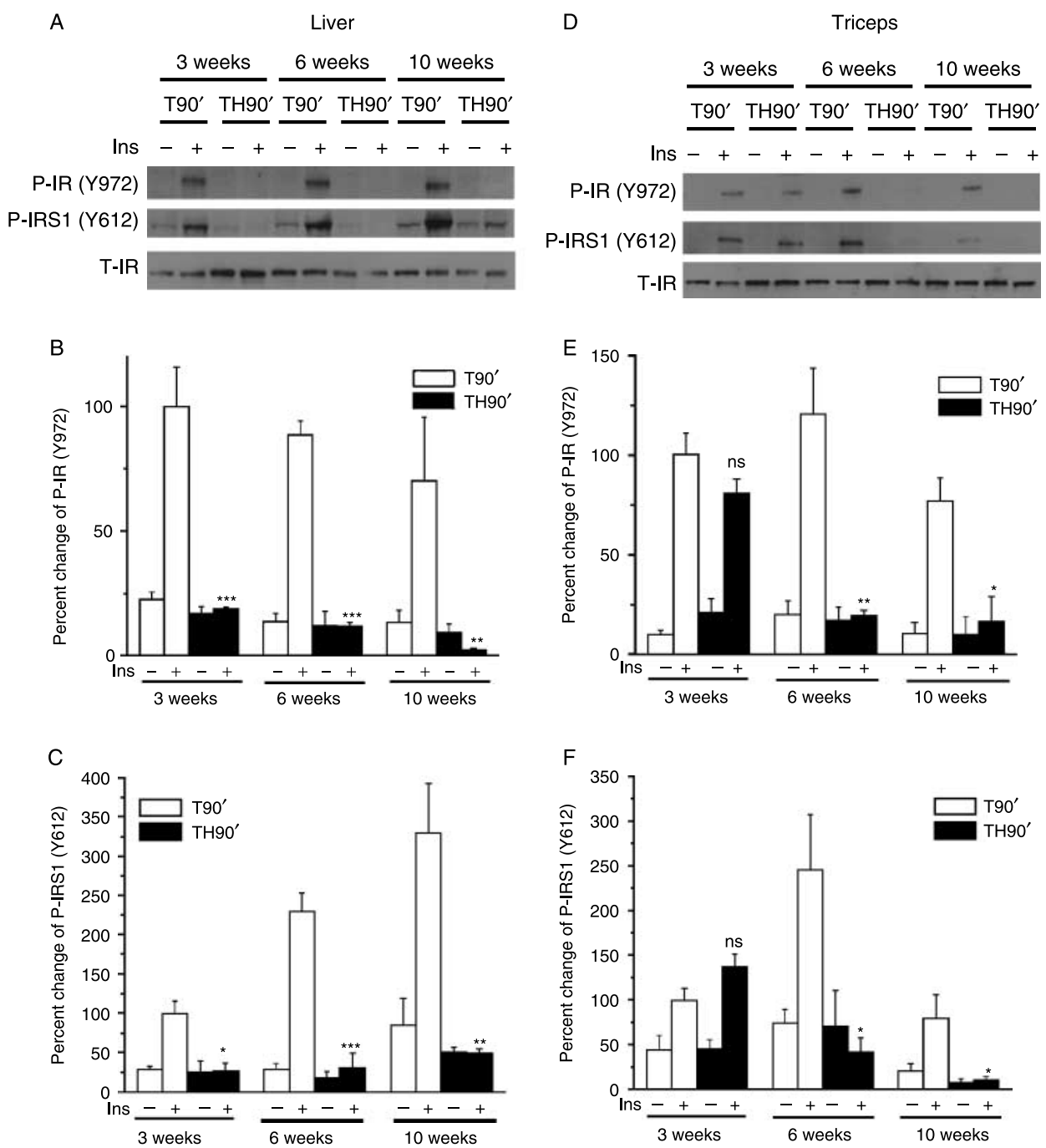

Figure 2 Hepatic and skeletal muscle insulin signaling via tyrosine phosphorylation of IRS- 1 and IR at different ages following trauma and hemorrhage. Following the same treatment procedure and time points described in Fig. 1, liver and triceps were removed and frozen in liquid nitrogen. (A and D) Tissue lysates from liver and triceps were subjected to western blotting with antibodies specific for phospho-insulin receptor (P-IR (Y972)), phospho-IRS1 (P-IRS1 (Y612)), and total insulin receptor (T-IR). (B, C, E, and F) Autoradiographs were quantified by scanning densitometry. Data are presented as the mean \pm S.E.M. of three rats in each group. 
Trauma and hemorrhage-induced decreases in insulin signaling (P-IR and P-IRS1): differences evident in triceps of 3-week old rats

Alterations of P-AKT (S473) may be due to changes in activation of upstream components of the insulin signaling pathway. To test this possibility, experiments were performed to determine the changes of activating tyrosine phosphorylation of IR and IRS1 in liver and skeletal muscle. Phosphorylation of tyrosine 972 of the IR (P-IR (Y972)) is known to be essential for the phosphorylation and binding of IRS1 (Backer et al. 1990, Biddinger \& Kahn 2006). IRS1 is necessary for transduction of the insulin signal, and phosphorylation of tyrosine 612 of IRS1 (P-IRS1 (Y612)) is critical for activation of the PI3K/ AKT pathway (Myers et al. 1992, Esposito et al. 2001). We therefore examined P-IR (Y972) and P-IRS1 (Y612) in both liver and triceps following trauma and hemorrhage in all age groups.

Our results indicate that insulin-induced P-IR (Y972) and P-IRS1 (Y612) were abolished in the liver by hemorrhage $\left(\mathrm{TH} 90^{\prime}\right)$ for all three ages examined (Fig. 2A-C). Similar to liver, phosphorylation of IR (Y972) and IRS1 (Y612) in skeletal muscle (triceps) was significantly decreased following trauma and hemorrhage in 6- and 10-week old rats. However, this did not occur in the 3-week old group where insulininduced P-IR and P-IRS was approximately the same following either trauma alone or following trauma and hemorrhage (Fig. 2D-F). Since the total amount of IR in liver and triceps was approximately the same in the three age groups (Fig. 2A and D), these results suggest that in skeletal muscle of 3-week old rats there is some protection from the trauma and hemorrhage-induced inhibition of IR signaling.

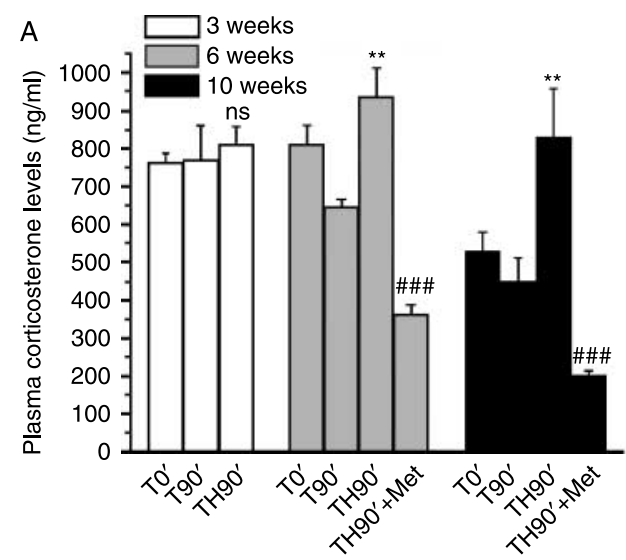

B
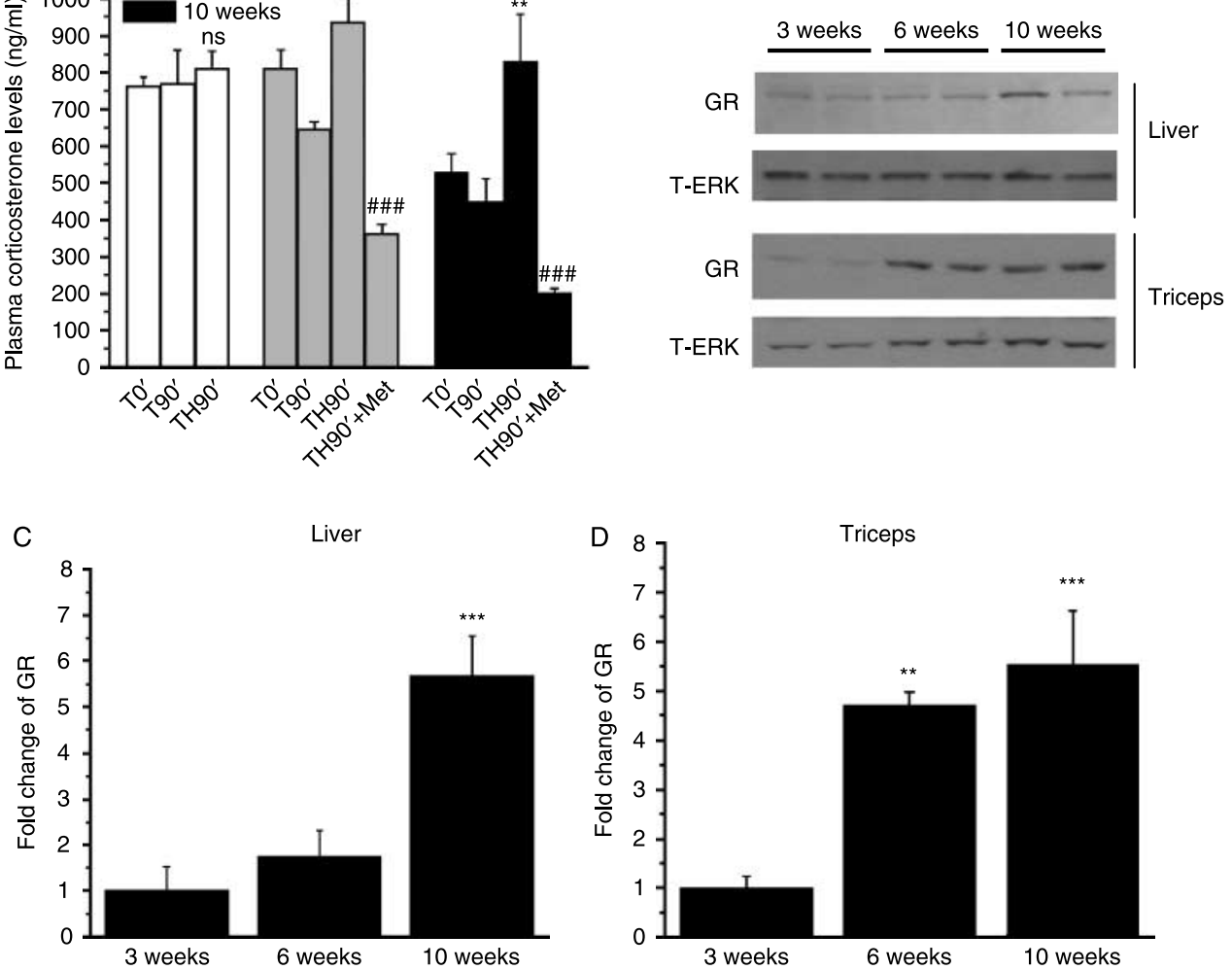

Figure 3 Corticosterone levels and expression of glucocorticoid receptor at different ages. (A) Rats were killed at the end of surgery $\left(\mathrm{TO}^{\prime}\right)$ in addition to the $\mathrm{T}^{\prime} \mathrm{O}^{\prime}$ and $\mathrm{TH} 90^{\prime}$ time points and blood was collected for the determination of plasma corticosterone levels. Results are expressed as ng corticosterone/ml plasma. In separate groups, metyrapone (Met) was administered as described in Materials and Methods. Each value represents the mean \pm S.E.M. of $5-6$ rats per group. ${ }^{* *} P<0 \cdot 01$, and ns, not significant, compared with $\mathrm{T}^{*} 0^{\prime}$ in the same age group. ${ }^{\sharp \#} P<0 \cdot 001$, compared with $\mathrm{TH}^{\sharp} 90^{\prime}$ in the same age group. (B) Following the same treatment procedure and time points described in Fig. 1, liver and triceps were removed and frozen in liquid nitrogen. Tissue lysates were subjected to western blotting with total glucocorticoid receptor (GR) and T-ERK antibody in liver and triceps. (C and D) Data are presented as the mean \pm s.E.M. of samples from 4-6 rats in each group with one set at the GR level in 3 -week old rats in liver $(C)$ and triceps (D). ${ }^{* *} P<0 \cdot 01$;

$* * * P<0 \cdot 001$; and ns, not significant, compared with 3 weeks group. 
Trauma and hemorrhage did not reduce $P-A K T$ in EDL of 3-week old rats

Previous studies in our lab reported that hemorrhageinduced acute insulin resistance was independent of muscle fiber type (Thompson et al. 2008). Since we have examined the effect of age on trauma and hemorrhage-induced insulin resistance in triceps, which is a type 1 (slow twitch) skeletal muscle, we next determined whether there was some protection against the development of hemorrhage-reduced insulin signaling in EDL, a type 2 (fast-twitch) skeletal muscle of 3-week old rats. Consistent with our findings in triceps, in 6- and 10- week old rats, insulin-induced P-AKT (S473) was greatly reduced following the combination of trauma and hemorrhage (TH90') in EDL (data not shown). However, as in triceps, insulin was still fully capable of inducing P-AKT (S473) in the EDL of 3-week old rats (data not shown).

Taken together, it is clear that at 3 weeks of age there is little or no development of acute skeletal muscle insulin resistance, in either slow-twitch (triceps) and fast-twitch (EDL) muscles following trauma and hemorrhage, whereas the acute development of insulin signaling defects still occurs in the liver.

\section{Corticosterone and GR levels at different ages and following trauma and hemorrhage}

Our previous studies indicate that glucocorticoids play a major role in skeletal muscle insulin resistance following trauma and hemorrhage in adult rats ( $\mathrm{Li}$ et al. 2009). To determine the role of glucocorticoids in hemorrhage-induced skeletal muscle insulin resistance at different ages, we first examined the levels of plasma corticosterone, the main plasma glucocorticoid in rodents. Corticosterone levels were not significantly altered 90 min following trauma $\left(\mathrm{T} 90^{\prime}\right)$ compared with trauma alone $\left(\mathrm{T}^{\prime}\right)$ in 3-, 6-, and 10-week groups, whereas corticosterone levels were significantly increased $90 \mathrm{~min}$ following trauma and hemorrhage $\left(\mathrm{TH} 90^{\prime}\right)$ at 6 and 10 weeks of age. There was a greater increase in 10-week old rats, due mainly to the lesser basal level of corticosterone. The higher basal corticosterone concentration of 6-week old rats was also evident in 3-week old rats. However, unlike the 6-week old rats, in the 3-week old rats there was no increase in corticosterone levels following the combination of trauma and hemorrhage (TH90'; Fig. 3A).

The amount of GR protein in liver and skeletal muscle was examined by western blotting and the levels were significantly higher in the liver of animals at 10 weeks of age than those at 3 and 6 weeks of age (Fig. 3B and C). In skeletal muscle
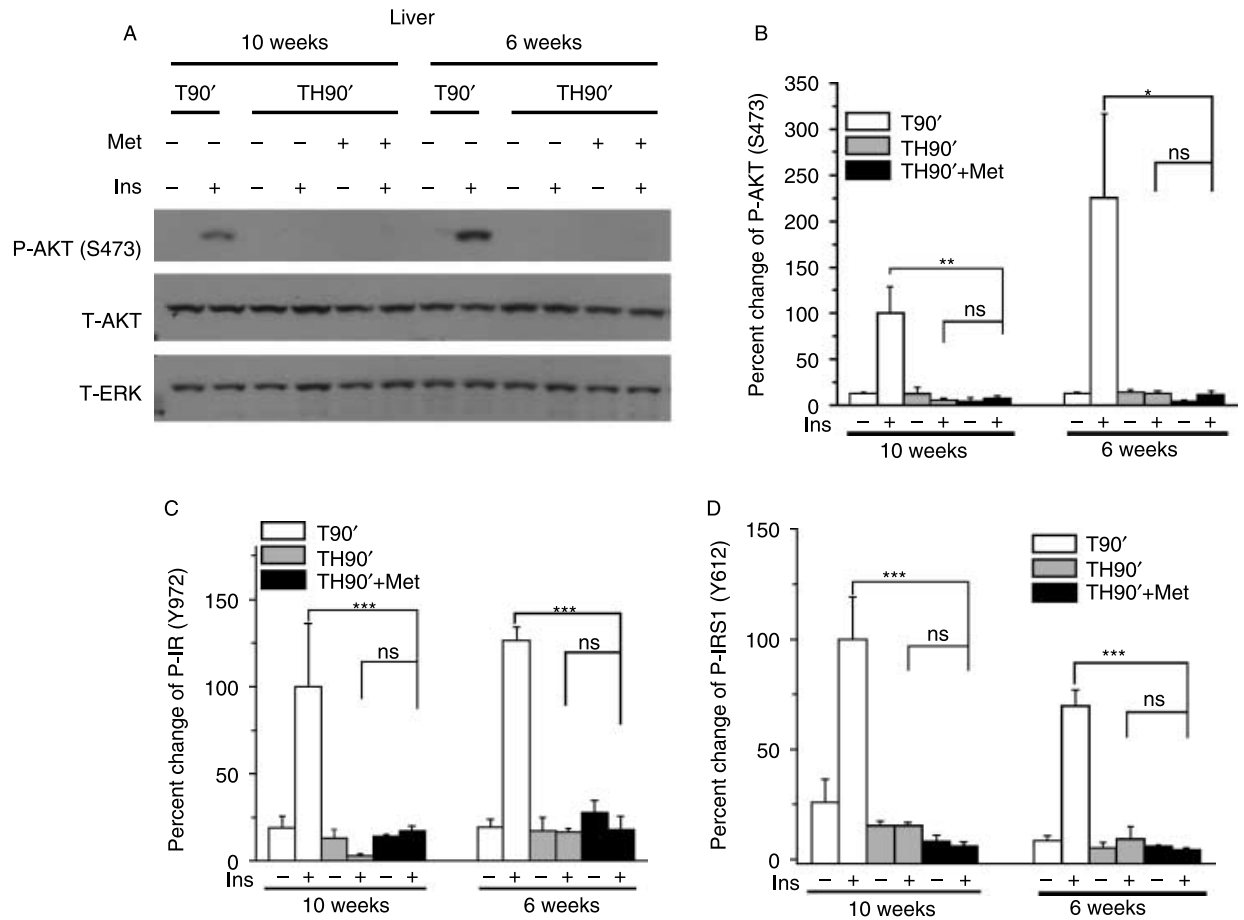

Figure 4 Decreasing corticosterone levels did not affect trauma and hemorrhage-induced insulin resistance in liver. Metyrapone (Met) was administered as described in Materials and Methods to 6- or 10-week old rats which were then subjected to trauma, or trauma and hemorrhage. Liver tissue lysates were subjected to western blotting with antibodies specific for P-AKT (S473), (P-IR (Y972)), P-IRS1 (Y612), T-AKT, and T-ERK. (A) A representative western blot. (B-D) Autoradiographs were quantified by scanning densitometry and presented as the mean \pm S.E.M. of three rats in each group and presented as the percentage change by insulin with the 10 -week old rats arbitrarily set to $100 \%$. 
(triceps), there was no difference in the total amount of GR between 6- and 10-week old rats which were both significantly higher than in 3-week old rats (Fig. 3B and D).

\section{Inhibition of corticosterone synthesis did not rescue hepatic insulin signaling following trauma and hemorrhage}

To further investigate the role of glucocorticoids in trauma and hemorrhage-induced insulin resistance, we pretreated animals with metyrapone an inhibitor of corticosterone synthesis, followed by determination of insulin resistance after trauma and hemorrhage. Previous work from our lab demonstrated that insulin signaling in control rats was not affected by metyrapone treatment ( $\mathrm{Li}$ et al. 2009). And, as expected, pretreatment with metyrapone, reduced the hemorrhage-induced increase of corticosterone levels in 10- and 6-week old rats (Fig. 3A). Since there was no increase of corticosterone following hemorrhage in 3-week old rats, metyrapone was not administered to these rats. Our previous data indicated that treatment with metyrapone did not change hepatic insulin resistance $60 \mathrm{~min}$ following hemorrhage in adult rats (Li et al. 2009). Similarly, we found that inhibition of corticosterone synthesis had no effect to protect the liver from developing insulin resistance following trauma and hemorrhage in 6- and 10-week old rats (Fig. 4A and B). In addition, the decrease of insulin-induced phosphorylation of IR (Y972) and IRS1 (Y612), the upstream insulin signaling molecules, was not prevented in the liver by pre-treatment with metyrapone (Fig. 4C and D). Therefore, these data further support our contention that glucocorticoids do not play an important role in the acute development of hepatic insulin resistance following hemorrhage.

\section{Inhibition of corticosterone synthesis prevented skeletal muscle insulin resistance following trauma and hemorrhage}

To determine whether glucocorticoids play a role in trauma and hemorrhage-induced insulin resistance in skeletal muscle, we measured phosphorylation of AKT (S473) following trauma and hemorrhage in rats pretreated with metyrapone. The ability of insulin to induce phosphorylation of AKT in triceps was significantly increased, such that the levels of $\mathrm{P}-\mathrm{AKT}$ (S473) in response to insulin were almost completely normalized by pre-treatment with metyrapone in 10 -week old rats (Fig. 5A and B). In addition, the ability of insulin to induce phosphorylation of IR (Y972) and IRS1 (Y612), which was
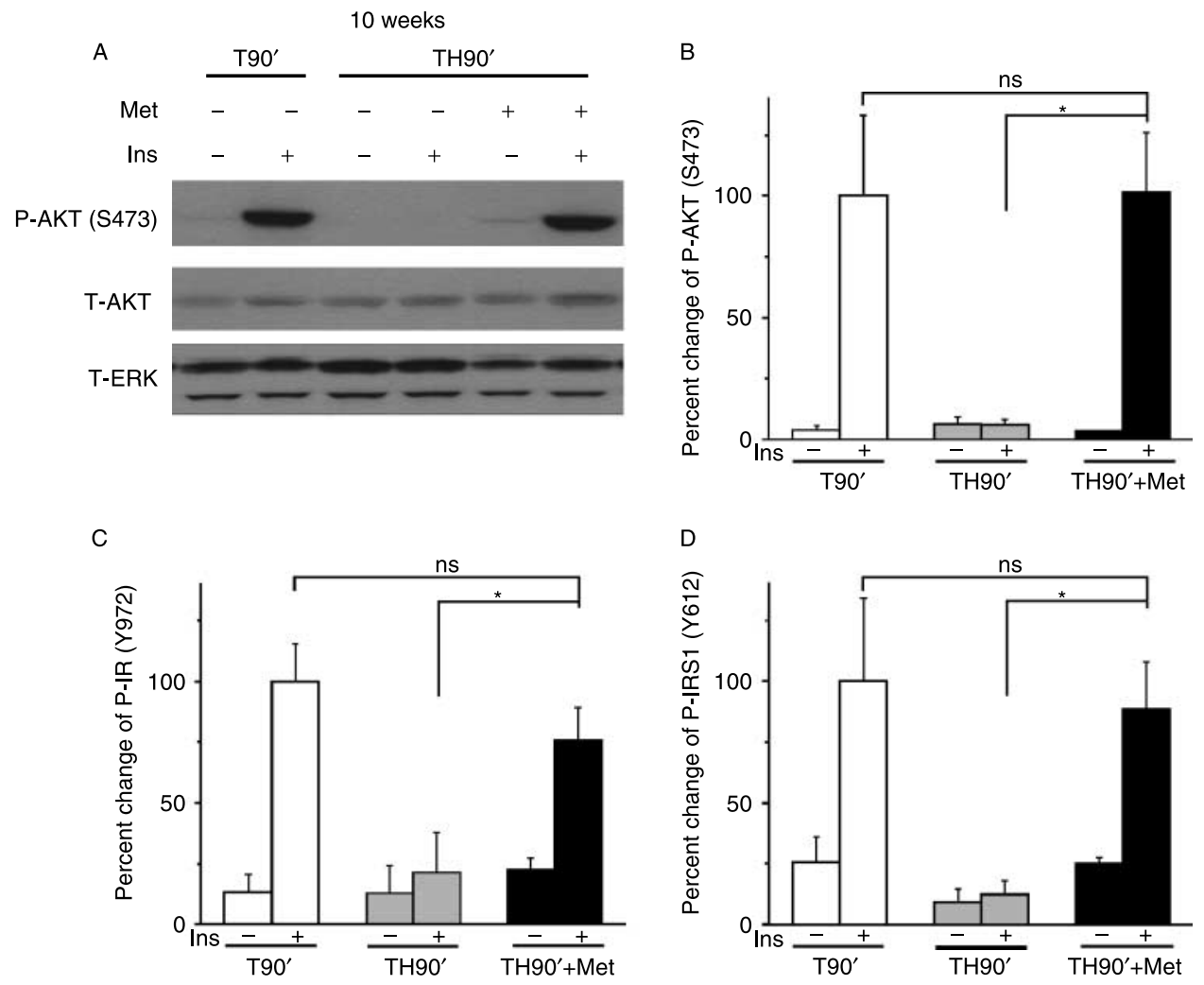

Figure 5 Effects of metyrapone on the impaired skeletal muscle insulin signaling in 10-week old rats. Following the same treatment procedures, time points and antibodies described in Fig. 4, triceps tissue lysates were subjected to western blotting. (A) A representative western blot. (B-D) Autoradiographs were quantified by scanning densitometry and presented as the mean \pm S.E.M. of 3-5 rats in each group and presented as the percentage change by insulin in the $\mathrm{T}^{\prime} 0^{\prime}$ group arbitrarily set to $100 \%$. 


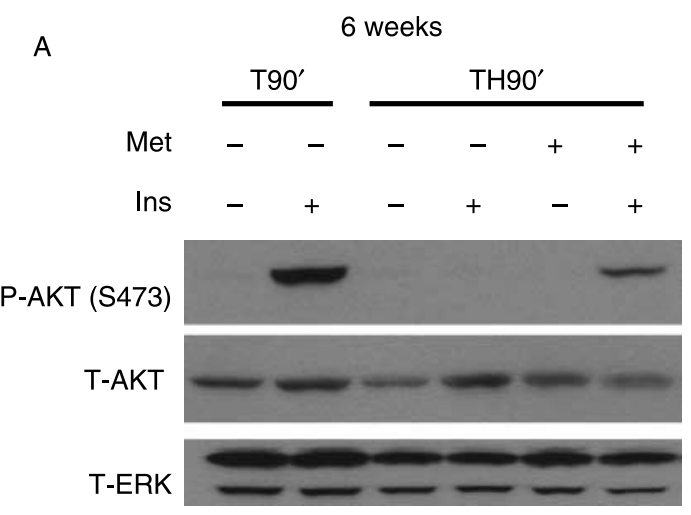

B

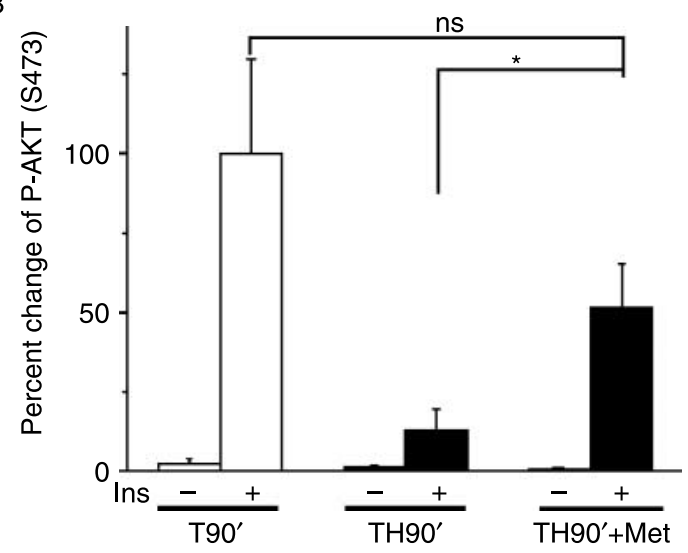

Figure 6 Effects of metyrapone on the impaired skeletal muscle insulin signaling in 6-week old rats. Following the same treatment procedures, time points and the P-AKT antibody described in Fig. 5, triceps tissue lysates were subjected to western blotting. (A) A representative western blot. (B) Autoradiographs were quantified by scanning densitometry and presented as the mean \pm S.E.M. of 3 rats in each group and presented as the percentage change by insulin in the T90' group arbitrarily set to $100 \%$.

negligible following trauma and hemorrhage in triceps, was also almost completely normalized by pretreatment with metyrapone (Fig. 5C and D). Pretreatment of 6-week old rats with metyrapone also partially restored phosphorylation of AKT (S473; Fig. 6A and B) in skeletal muscle. Together, these data demonstrate that the acute development of insulin resistance in skeletal muscle following trauma and hemorrhage can be inhibited by blocking glucocorticoid synthesis. Again, since there was no increase of corticosterone following hemorrhage in 3-week old rats, and since there was no development of insulin resistance in skeletal muscle of 3-week old rats, the effects of metyrapone were not studied.

\section{Discussion}

Hyperglycemia is a risk factor and associated with increased morbidity and mortality following injury, infection and critical illness in children and adults (Cochran et al. 2003,
Krinsley 2004, Branco et al. 2005, Garcia et al. 2007, Klein et al. 2007). This hyperglycemia is associated with insulin resistance, but little is known about whether this insulin resistant state develops at different ages. Since glucose production by the liver and glucose uptake by skeletal muscle are important in regulating glucose levels, interruption of insulin signaling in liver and skeletal muscle can cause hyperglycemia. Our previous findings indicate that acute insulin resistance in liver and skeletal muscle occurs in adult rats following trauma and hemorrhage (Ma et al. 2003, 2004, Thompson et al. 2008, Xu et al. 2008, Li et al. 2009). In the present study, we used rats of various ages to determine if hepatic and skeletal muscle insulin resistance occurs at younger ages.

The rapid development of a defect of insulin signaling via the IR/IRS-1/PI3K/AKT pathway in liver and skeletal muscle, what we call acute insulin resistance, was observed following trauma and hemorrhage in livers of 3-, 6-, and 10-week old rats, and in skeletal muscle from $6-$ and 10-week old rats. However, we did not observe the development of acute insulin resistance in skeletal muscle of 3-week old rats following trauma and hemorrhage. Measurement of insulin-induced AKT serine phosphorylation as well as tyrosine phosphorylation of IR and IRS1 were completely consistent. Phosphorylation of IR, IRS1, and AKT decreased rapidly in liver and skeletal muscle of 6- and 10-week old rats, and in the liver of 3-week old rats, following trauma and hemorrhage. However, there was no change in the skeletal muscle of 3-week old rats in response to trauma and hemorrhage. In human studies, insulin resistance in critically ill children may be due to down regulation of IRs (Xu et al. 1996). However, due to the rapidity of the response to trauma and hemorrhage in the present studies, IR content did not change in either liver or skeletal muscle.

Skeletal muscle is a mixture of two fiber types, type 1 (slow-twitch) and type 2 (fast-twitch) fibers (Schiaffino \& Serrano 2002). The response of slow-twitch skeletal muscle to insulin is greater than fast-twitch skeletal muscle (James et al. 1985). We found that trauma and hemorrhage-induced acute insulin resistance did not occur in triceps (slow-twitch) muscle or in EDL (fast-twitch) muscle at 3 weeks, but did at 6 and 10 weeks of age in both muscle types. These results indicate that there is no muscle type specificity in the agerelated acute development of insulin resistance following trauma and hemorrhage in skeletal muscles (also see Thompson et al. (2008)).

Adrenal glucocorticoid content differs during rat development (Morii \& Huggins 1962), and GRs levels differ among tissues (Chrousos \& Kino 2005). Glucocorticoids levels increase dramatically following injury or infection (Vaughan et al. 1982, Li et al. 2009). Our earlier work implicates glucocorticoids as a causative factor in skeletal muscle insulin resistance following trauma and hemorrhage in adult rats ( $\mathrm{Li}$ et al. 2009). Therefore, increased glucocorticoid synthesis or differences in GR 
levels in the liver and skeletal muscle could play a role in the acute development of insulin resistance following trauma and hemorrhage. Corticosterone levels increased rapidly following hemorrhage in $10-$ week old rats. Basal glucocorticoid levels in 3- and 6-week old rats were already as high as the concentration attained in the 10-week old rats following trauma and hemorrhage. However, corticosterone levels increased further only in the 6-week old rats, not changing at all in the 3 -week old rats. This indicates that the 3-week old rats do not respond to the stress of trauma and hemorrhage as in 6- and 10-week old rats, but are capable of secreting high basal levels of corticosterone. A likely reason for the lack of an increase in corticosterone following trauma and hemorrhage in 3-week old rats is the lesser sensitivity of the hypothalamic-pituitary-adrenal axis to stress in young rats (Leal et al. 1999, Yoshimura et al. 2003). The response of 6-week old rats was more modest than in 10-week old rats, but there was an increase of corticosterone in response to trauma and hemorrhage.

We also observed that skeletal muscle GR levels in 3-week old rats were one-forth or lower than in triceps of 6- and 10 -week old rats. Thus, with little or no increase of corticosterone levels following trauma and hemorrhage, and with the low levels of triceps GRs, there is a much lower potential for a response of skeletal muscle of 3-week old rats to glucocorticoids as compared to adult rats ( $\mathrm{Li}$ et al. 2009). Metyrapone decreases corticosterone synthesis levels by inhibiting 11- $\beta$ hydroxylation of desoxyxoticosterone (Metcalf \& Beaven 1968, Schoneshofer et al. 1979), and can lead to increased adrenal levels of corticosterone and aldosterone precursors (Metcalf \& Beaven 1968, Taylor \& Fishman 1988, Aupetit-Faisant 1990) which may present a problem if used chronically. In skeletal muscle of 6- and 10-week old rats, which express much higher levels of GRs, the acute inhibition of glucocorticoid synthesis by metyrapone resulted in at least a partial rescue of insulin signaling following trauma and hemorrhage. Thus, we hypothesize that the large increase in skeletal muscle GR levels between 3 weeks and 6 or 10 weeks of age results in the ability of the skeletal muscle to respond to the increased glucocorticoid levels following trauma and hemorrhage, resulting in the acute development of insulin resistance. However, it is not known whether glucocorticoids play a similar role in the defects of muscle insulin signaling induced by the other stresses (Wu et al. 2004, Lang \& Frost 2007). In the liver, the level of GRs is irrelevant since development of acute hepatic insulin resistance is not dependent upon glucocorticoids (Thompson et al. 2008, Xu et al. 2008, Li et al. 2009).

In summary, hepatic insulin resistance following trauma and hemorrhage occurs at all age rats tested, even just after weaning, and involves multiple components in the insulin signaling pathway. Insulin resistance does not develop in either slow-twitch (triceps) or fast-twitch (EDL) skeletal muscle following trauma and hemorrhage in 3-week old rats, and the lack of response to trauma and hemorrhage may be due to the low levels of GR in the skeletal muscle of young rats. As the rats age, they develop the ability to respond to trauma and hemorrhage with acute skeletal muscle insulin resistance that is glucocorticoid-dependent.

\section{Declaration of interest}

The authors declare that there is no conflict of interest that could be perceived as prejudicing the impartiality of the research reported.

\section{Funding}

This research is supported by grants from the National Institutes of Health Grant (DK62071) and the Veterans Administration Merit Review to J L M.

\section{Acknowledgements}

We thank the University of Alabama at Birmingham Clinical Nutrition Research Center for corticosterone measurements (Center grant P30 DK56336). We also would like to thank J L Franklin, Drs V William, J Xu, N A Kokorina, and H T Kim, for their helpful and insightful discussions and suggestions.

\section{References}

Alexander CM 2003 The coming of age of the metabolic syndrome. Diabetes Care 26 3180-3181.

Arabi YM, Dabbagh OC, Tamim HM, Al-Shimemeri AA, Memish ZA, Haddad SH, Syed SJ, Giridhar HR, Rishu AH, Al-Daker MO et al. 2008 Intensive versus conventional insulin therapy: a randomized controlled trial in medical and surgical critically ill patients. Critical Care Medicine $\mathbf{3 6}$ 3190-3197.

Aupetit-Faisant B 1990 Plasma levels of aldosterone precursors (including the 18-hydroxylated compounds in this synthesis pathway) - a tool for the analysis of adrenal disorders? Hormone Research 34 142-150.

Backer JM, Kahn CR, Cahill DA, Ullrich A \& White MF 1990 Receptormediated internalization of insulin requires a 12 -amino acid sequence in the juxtamembrane region of the insulin receptor beta-subunit. Journal of Biological Chemistry 265 16450-16454.

Van den Berghe G, Wouters P, Weekers F, Verwaest C, Bruyninckx F, Schetz M, Vlasselaers D, Ferdinande P, Lauwers P \& Bouillon R 2001 Intensive insulin therapy in the critically ill patients. New England Journal of Medicine 345 1359-1367.

Biddinger SB \& Kahn CR 2006 From mice to men: insights into the insulin resistance syndromes. Annual Review of Physiology 68 123-158.

Bochicchio GV, Sung J, Joshi M, Bochicchio K, Johnson SB, Meyer W \& Scalea TM 2005 Persistent hyperglycemia is predictive of outcome in critically ill trauma patients. Journal of Trauma $\mathbf{5 8}$ 921-924.

Braithwaite SS 2009 Glucose control in critically ill patients. New England Journal of Medicine 361 89-90.

Branco RG, Garcia PC, Piva JP, Casartelli CH, Seibel V \& Tasker RC 2005 Glucose level and risk of mortality in pediatric septic shock. Pediatric Critical Care Medicine 6 470-472.

Carter EA 1998 Insulin resistance in burns and trauma. Nutrition Reviews $\mathbf{5 6}$ S170-S176.

Chrousos GP \& Kino T 2005 Intracellular glucocorticoid signaling: a formerly simple system turns stochastic. Science's STKE 2005 e48.

Cochran A, Scaife ER, Hansen KW \& Downey EC 2003 Hyperglycemia and outcomes from pediatric traumatic brain injury. Journal of Trauma $\mathbf{5 5}$ 1035-1038. 
Cree MG \& Wolfe RR 2008 Postburn trauma insulin resistance and fat metabolism. American Journal of Physiology. Endocrinology and Metabolism 294 E1-E9.

Esposito DL, Li Y, Cama A \& Quon MJ 2001 Tyr(612) and Tyr(632) in human insulin receptor substrate-1 are important for full activation of insulinstimulated phosphatidylinositol 3-kinase activity and translocation of GLUT4 in adipose cells. Endocrinology 142 2833-2840.

Finfer S, Chittock DR, Su SY, Blair D, Foster D, Dhingra V, Bellomo R, Cook D, Dodek P, Henderson WR et al. 2009 Intensive versus conventional glucose control in critically ill patients. New England Journal of Medicine 360 1283-1297.

Garcia BR, Tasker RC, Ramos Garcia PC, Piva JP \& Dias XL 2007 Glycemic control and insulin therapy in sepsis and critical illness. Journal of Pediatrics $\mathbf{8 3}$ S128-S136.

Giorgino F, Almahfouz A, Goodyear LJ \& Smith RJ 1993 Glucocorticoid regulation of insulin receptor and substrate IRS-1 tyrosine phosphorylation in rat skeletal muscle in vivo. Journal of Clinical Investigation 91 2020-2030.

Hansen TK, Thiel S, Wouters PJ, Christiansen JS \& Van den Berghe G 2003 Intensive insulin therapy exerts antiinflammatory effects in critically ill patients and counteracts the adverse effect of low mannose-binding lectin levels. Journal of Clinical Endocrinology and Metabolism 88 1082-1088.

Hill MM, Feng J \& Hemmings BA 2002 Identification of a plasma membrane Raft-associated PKB Ser473 kinase activity that is distinct from ILK and PDK1. Current Biology 12 1251-1255.

Ikezu T, Okamoto T, Yonezawa K, Tompkins RG \& Martyn JA 1997 Analysis of thermal injury-induced insulin resistance in rodents. Implication of postreceptor mechanisms. Journal of Biological Chemistry 272 25289-25295.

James DE, Jenkins AB \& Kraegen EW 1985 Heterogeneity of insulin action in individual muscles in vivo: euglycemic clamp studies in rats. American Journal of Physiology 248 E567-E574.

Klein GW, Hojsak JM \& Rapaport R 2007 Hyperglycemia in the pediatric intensive care unit. Current Opinion in Clinical Nutrition and Metabolic Care $\mathbf{1 0}$ 187-192.

Krinsley JS 2004 Effect of an intensive glucose management protocol on the mortality of critically ill adult patients. Mayo Clinic Proceedings 79 992-1000.

Lang CH \& Frost RA 2007 Sepsis-induced suppression of skeletal muscle translation initiation mediated by tumor necrosis factor alpha. Metabolism: Clinical and Experimental 56 49-57.

Langouche L, Vanhorebeek I \& Van den Berghe G 2005 The role of insulin therapy in critically ill patients. Treatments in Endocrinology 4 353-360.

Leal AM, Carvalho J \& Moreira AC 1999 Ontogenetic diurnal variation of adrenal responsiveness to $\mathrm{ACTH}$ and stress in rats. Hormone Research $\mathbf{5 2}$ 25-29.

Li L, Thompson LH, Zhao L \& Messina JL 2009 Tissue specific difference in the molecular mechanisms for the development of acute insulin resistance following injury. Endocrinology 150 24-32.

Ma Y, Wang P, Kuebler JF, Chaudry IH \& Messina JL 2003 Hemorrhage induces the rapid development of hepatic insulin resistance. American Journal of Physiology. Gastrointestinal and Liver Physiology 284 G107-G115.

Ma Y, Toth B, Keeton AB, Holland LT, Chaudry IH \& Messina JL 2004 Mechanisms of hemorrhage-induced hepatic insulin resistance: role of tumor necrosis factor-alpha. Endocrinology 145 5168-5176.

McEniery CM, Wilkinson IB \& Avolio AP 2007 Age, hypertension and arterial function. Clinical and Experimental Pharmacology and Physiology 34 $665-671$.

Meneilly GS \& Tessier D 1995 Diabetes in the elderly. Diabetic Medicine 12 949-960.

Metcalf MG \& Beaven DW 1968 The metopirone test of pituitary corticotrophin release. Evaluation of 101 tests. American Journal of Medicine 45 176-186.
Morii S \& Huggins C 1962 Adrenal apoplexy induced by 7,12dimethylbenz(A)anthracene related to corticosterone content of adrenal gland. Endocrinology 71 972-976.

Myers MG Jr, Backer JM, Sun XJ, Shoelson S, Hu P, Schlessinger J, Yoakim M, Schaffhausen B \& White MF 1992 IRS-1 activates phosphatidylinositol $3^{\prime}$-kinase by associating with src homology 2 domains of p85. PNAS 89 10350-10354.

Nakae J \& Accili D 1999 The mechanism of insulin action. Journal of Pediatric Endocrinology and Metabolism 12 721-731.

Qi D \& Rodrigues B 2007 Glucocorticoids produce whole body insulin resistance with changes in cardiac metabolism. American Journal of Physiology. Endocrinology and Metabolism 292 E654-E667.

Saad MJA, Folli F, Kahn JA \& Kahn CR 1993 Modulation of insulin receptor, insulin receptor substrate-1, and phosphatidylinositol 3-kinase in liver and muscle of dexamethasone-treated rats. Journal of Clinical Investigation 92 2065-2072.

Schiaffino S \& Serrano A 2002 Calcineurin signaling and neural control of skeletal muscle fiber type and size. Trends in Pharmacological Sciences $\mathbf{2 3}$ 569-575.

Schoneshofer M, Schefzig B \& Oelkers W 1979 Evidence of adrenal 18-hydroxylase inhibition by metyrapone in man. Hormone and Metabolic Research 11 306-308.

Taylor AL \& Fishman LM 1988 Corticotropin-releasing hormone. New England Journal of Medicine 319 213-222.

Thompson LH, Kim HT, Ma Y, Kokorina N \& Messina JL 2008 Acute muscle-type specific insulin resistance following injury. Molecular Medicine 11-12 715-723.

Vaughan GM, Becker RA, Allen JP, Goodwin CW Jr, Pruitt BA Jr \& Mason AD Jr 1982 Cortisol and corticotrophin in burned patients. Journal of Trauma 22 263-273.

Vegiopoulos A \& Herzig S 2007 Glucocorticoids, metabolism and metabolic diseases. Molecular and Cellular Endocrinology 275 43-61.

White MF 1997 The insulin signalling system and the IRS proteins. Diabetologia 40 S2-S17.

Witchel SF \& DeFranco DB 2006 Mechanisms of disease: regulation of glucocorticoid and receptor levels - impact on the metabolic syndrome. Nature Clinical Practice. Endocrinology \& Metabolism 2 621-631.

Wu Y, Barrett EJ, Long W \& Liu Z 2004 Glucocorticoids differentially modulate insulin-mediated protein and glycogen synthetic signaling downstream of protein kinase B in rat myocardium. Endocrinology 145 1161-1166.

Xu F, Yang X, Lu Z \& Kuang F 1996 Evaluation of glucose metabolic disorder: insulin resistance and insulin receptors in critically ill children. Chinese Medical Journal 109 807-809.

Xu J, Kim HT, Ma Y, Zhao L, Zhai L, Kokorina N, Wang P \& Messina JL 2008 Trauma and hemorrhage-induced acute hepatic insulin resistance: dominant role of tumor necrosis factor (TNF)-alpha. Endocrinology 149 2369-2382.

Yoshimura S, Sakamoto S, Kudo H, Sassa S, Kumai A \& Okamoto R 2003 Sex-differences in adrenocortical responsiveness during development in rats. Steroids 68 439-445.

Received in final form 7 September 2009

Accepted 14 September 2009

Made available online as an Accepted Preprint 14 September 2009 